\title{
Proposed criteria for the identification of polycystic ovary syndrome following menopause: An ancillary study of the Brazilian Longitudinal Study of Adult Health (ELSA-Brasil) is
}

\author{
Ligia Gabrielli $^{\mathrm{a}, \mathrm{b}, *}$, Maria da Conceição Chagas de Almeida ${ }^{\mathrm{a}, \mathrm{c}}$, Estela M.L. Aquino ${ }^{\mathrm{a}}$ \\ a Institute of Collective Health, Federal University of Bahia, Rua Basílio da Gama, s/n, Campus Universitário do Canela, 40110-040 Salvador, Bahia, Brazil \\ ${ }^{\mathrm{b}}$ Bahia State Center for Diabetes and Endocrinology, Av. ACM, s/n, Iguatemi, 40275-350 Salvador, Bahia, Brazil

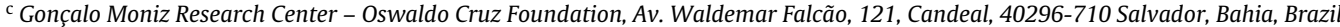

\section{A R T I C L E I N F O}

\section{Article history:}

Received 28 September 2014

Received in revised form 12 March 2015

Accepted 25 April 2015

\section{Keywords:}

Polycystic ovary syndrome

Menopause

Insulin resistance

\begin{abstract}
A B S T R A C T
Objectives: To propose plausible criteria with which to identify menopausal women with PCOS.

Study design: A cross-sectional study involving the baseline data of 713 menopausal women at admission to the Brazilian Longitudinal Study of Adult Health (ELSA-Brasil) in Salvador, Bahia, Brazil.

Main outcome measures: PCOS was identified by the presence of two of three criteria. (1)A history of amenorrhea or oligomenorrhea (OL) (regular intermenstrual intervals $\geq 35$ days during reproductive life); (2) clinical or biochemical hyperandrogenism (HA), identified by a score $\geq 5$ points in a hirsutism questionnaire constructed and validated for women in this age group, or total or free testosterone $\geq$ the 95th percentile for women considered normal; (3) insulin resistance (IR) (a homeostatic model assessment [HOMA] index $\geq 2.2$ ). Validation was performed using probable epidemiological endpoints.

Results: According to these criteria, 7.6\% of the women in the sample had PCOS. Of these, $7.4 \%$ had HA and OL, 72.2\% had HA and IR, 14.8\% had OL and IR and 5.6\%, had HA, OL and IR. Women with PCOS were younger, had had fewer pregnancies and entered menopause earlier. Positive associations were found between PCOS and overweight (PR: 1.31; 95\%CI: 1.18-1.46), obesity (1.44; 1.01-2.06), carbohydrate metabolism disorders (impaired glucose tolerance and diabetes mellitus) $(1.30 ; 1.03-1.65)$, and with diabetes alone $(1.41 ; 0.83-2.39)$, although this latter association failed to reach statistical significance.

Conclusion: The women selected in accordance with these criteria had the characteristics of PCOS that are not only expected, but also widely associated with this disorder.
\end{abstract}

(C) 2015 Elsevier Ireland Ltd. All rights reserved.

\section{Introduction}

Polycystic ovary syndrome (PCOS) is the most common endocrinopathy in women of reproductive age, with a prevalence that ranges from 2 to 15\% [1,2]. Oligoovulation, hyperandrogenism and ovaries with a polycystic appearance characterize the condition. Strongly associated with obesity and type 2 diabetes mellitus (T2DM), its association with dyslipidemia and arterial hypertension is positive albeit less intense. On the other hand, the syndrome has been associated with diverse cardiovascular risk factors [3] and with subclinical vascular disease [4].

\footnotetext{
is This study was conducted at the Federal University of Bahia's Research Center for the ELSA-Brasil Longitudinal Study, Salvador, Bahia, Brazil.

* Corresponding author at: Rua Basílio da Gama, s/n, Campus Universitário do Canela, 40110-040 Salvador, Bahia, Brazil. Tel.: +55 7132837422.

E-mail address: ligiagabrielli@uol.com.br (L. Gabrielli).
}

Nevertheless, demonstrating an increase in the occurrence of cardiovascular events in women with PCOS has proven difficult, with various hypotheses having been formulated to explain this apparent paradox. First, these events usually occur after menopause, a time at which identifying PCOS is no easy task. The cessation of menstruation and a reduction in hyperandrogenism and in the polycystic appearance of the ovaries, in addition to the inexistence of validated and established standards with which to evaluate serum testosterone levels or to quantify body hair in menopausal women makes diagnosis of the syndrome at this time of life difficult $[3,5]$. Secondly, improvements in the syndrome in the final reproductive years may contribute toward reducing these women's cardiovascular risk [6]. Regular menstruation and fertility after 35 years of age is not uncommon, sometimes accompanied by a reduction in hyperandrogenism $[7,8]$. A third explanation may concern the existence of a protective factor inherent to these women $[5,9]$ that could diminish the risk factors associated with this condition. Nevertheless, some features of the syndrome have 
been shown to remain after menopause. The high serum testosterone levels and the polycystic morphology of the ovaries serve as markers of a risk of coronary disease or an increase in the extent of coronary disease $[10,11]$. Higher testosterone levels are common in menopausal women with PCOS whose ovaries continue to have a polycystic appearance [12].

Only one study on the prevalence of PCOS in the menopause was identified [5]. That paper reported a rate of PCOS of $6.7 \%$ in a small sample of unselected women using the Rotterdam criteria, which are recommended for women of reproductive age [3]. To avoid using diagnostic criteria defined specifically for younger women and aiming at incorporating characteristics related to the physiopathology of the syndrome into the diagnosis, a group of investigators proposed and tested what they refer to as a putative phenotype for PCOS in the menopause. Those investigators reported an association between some components of this phenotype and cardiovascular disease and the distinct hormone profile expected in women with PCOS $[13,14]$.

For women of reproductive age, the Androgen Excess Society recommends including hyperandrogenism as a basic component for a diagnosis of PCOS [15]. The phenotypes proposed consist of a combination of hyperandrogenism, menstrual dysfunction and ovaries with polycystic morphology, with those investigators suggesting that diagnosis of the syndrome should potentially signal the risk of insulin resistance (IR) and its resulting metabolic abnormalities. Those who oppose the inclusion of hyperandrogenism as an obligatory diagnostic criterion argue that phenotypes without this component may equally predispose to IR and its consequences [16]. Although not yet obligatory in defining PCOS in women of reproductive age, IR, in addition to being an important pathophysiological element, has been taken into consideration when phenotypes for the identification of the syndrome are proposed [13,17].

Until long-term longitudinal studies are conducted to monitor women with PCOS, estimates of the risk of cardiovascular events in these women can only be made by identifying the diagnostic criteria appropriate for the postmenopausal phase. Since not all women with PCOS are diagnosed during their reproductive life, [18] identifying them at any stage in their lives may raise awareness of the risks to which they are exposed, enabling specific recommendations to be made and healthcare interventions to be implemented, with subsequent consequences for prognosis [3].

The aim of the present study was to propose and validate plausible criteria for identifying postmenopausal women with PCOS that could be used within the context of epidemiological research.

\section{Methods}

\subsection{Study population}

This study was conducted with female staff members of the Federal University of Bahia, one of the centers included in the Longitudinal Adult Health Study (ELSA-Brasil) [19]. ELSA-Brasil involves a total of 8212 women and 6893 men recruited at six different centers. The objective was to form a cohort that would be monitored through telephone interviews and periodic visits to the study centers for further exams and interviews. T2DM and cardiovascular disease were among the principal endpoints of the ELSA-Brasil study, and the data accumulated from this study now form a major research platform.

\subsection{Criteria proposed}

Criteria were proposed based on the assumption that androgenic manifestations and menstrual disturbances constitute essential elements by which to diagnose the syndrome and also on the pathophysiological importance of IR, a component expected to be common in women with PCOS in the age range of interest and that predisposes to cardiometabolic abnormalities. The study then proposed to identify PCOS in any menopausal women with two of the following three features: (a) clinical or biochemical hyperandrogenism; (b) menstrual dysfunction; and (c) IR. In this way, four phenotypes, all, in principle, considered plausible, were identified, as follows:
1. Hyperandrogenism

2. Hyperandrogenism

3. Oligo/amenorrhea

4. Hyperandrogenism
Oligo/amenorrhea

Insulin resistance

Insulin resistance

Oligo/amenorrhea
Insulin resistance

Oligo/amenorrhea was defined as a customary intermenstrual period $\geq 35$ days. Hyperandrogenism was defined as a score $\geq 5$ in the Hirsutism Questionnaire (Hirsuta), validated for use in this population [20] or total or free testosterone levels at or above the 95th percentile of the women in this study who were menopausal, not oligomenorrheic, not infertile and who had no known previous diagnosis of PCOS. IR was considered to be present in women with a homeostasis model assessment (HOMA) index $\geq 2.2$, obtained using the formula: fasting insulin $(\mathrm{mU} / \mathrm{l}) \times$ fasting glucose $(\mathrm{mmol} / \mathrm{l}) / 22.5$ [21]. This cut off point was defined for this study population following evaluation of the receiver operating characteristic (ROC) curve in which the results of the glucose tolerance test and the HOMA index were compared (sensitivity 60\%, specificity $71.1 \%$, correctly classified tests $70 \%$ and overall performance of the test 0.7256 ). This value corresponds to the 75 th percentile of the non-obese and non-diabetic study population [22].

With the objective of comparing the group identified using the proposed criteria with women of reproductive age with PCOS, premenopausal women with both menstrual dysfunction and hyperandrogenism, defined according to the same criteria described above using total and free testosterone levels, were identified and the 95th percentile was calculated for this group of women. This selection process is in agreement with the criteria recommended by the National Institutes of Health in 1990.

\subsection{Validation of the criteria}

The proposed criteria were applied to the menopausal women with these phenotypes identified at admission to the ELSA-Brasil study. Women were considered menopausal if they had been amenorrheic for at least a year and had follicle-stimulating hormone (FSH) levels $\geq 25 \mathrm{mIU} / \mathrm{ml}$. Women currently in use of oral contraceptives or testosterone were excluded from the study. None of the participants was in use of corticosteroids.

To validate these criteria, the case group was compared with the non-case group with respect to the occurrence of probable outcomes such as overweight, obesity and disorders of carbohydrate metabolism, i.e. glucose intolerance together with type 2 diabetes mellitus (T2DM) or T2DM alone.

Overweight was defined as body mass index (BMI) $\geq 25$ and obesity as $\mathrm{BMI} \geq 30$. T2DM was defined as being present in women: (a) reporting diabetes, in use of diabetes drugs, with two abnormal tests; (b) either reporting diabetes or in use of drugs, with at least one abnormal test; or (c) reporting diabetes, in use of drugs, with normal tests. In addition, T2DM was considered to be present in those women with (d) no previous diagnosis, not using drugs, but with at least two abnormal tests. Women with a history of type 1 diabetes were excluded from the study. For a diagnosis of T2DM, fasting glucose $>126 \mathrm{mg} / \mathrm{dl}$, glycated hemoglobin $\geq 6.5 \%$ and a glucose tolerance test $>200 \mathrm{mg} / \mathrm{dl}$ at $120 \mathrm{~min}$ were required [23]. This same test was used to identify women with glucose 
intolerance, which was defined as glucose levels $>140$ and $<200 \mathrm{mg} / \mathrm{dl}$ at $120 \mathrm{~min}$ after a 75 -g oral glucose load [24].

\subsection{Biochemical tests}

Blood samples were centrifuged for $30 \mathrm{~min}$ and the separated serum was stored at $-80^{\circ} \mathrm{C}$ until hormone measurement. Fasting glucose, 2-h glucose following a 75-g glucose load and insulin testing were performed in plasma samples. FSH, luteinizing hormone (LH) and sex hormone-binding globulin (SHBG) were measured using a chemiluminescence immunoassay technique (Immulite 2000, Siemens Healthcare Diagnostics Products, Llamberis, United Kingdom). Total testosterone was also measured using a chemiluminescence immunoassay (Architect 2000, Abbott Laboratories, IL, USA). Free testosterone levels and the free androgen index were calculated based on testosterone and SHBG levels, using the Vermeulen equation for the former and the formula $(\mathrm{T} / \mathrm{SHBG}) \times 100$ (both in $\mathrm{nmol} / \mathrm{l}$ ) for the latter.

\subsection{Data analysis}

In accordance with the normality of distribution, means or medians were calculated with measures of dispersion (standard deviation [SD] or interquartile range [IQR]), and Student's $t$-test or the Mann-Whitney test was used. Some variables were classified using traditional cut-off points or by taking the median calculated from the data in the present study into consideration. When it was not possible to use the chi-square test, this was substituted by Fisher's exact test, as appropriate. Some variables with more than two categories were analyzed using Cuzick's test for trend.

To study the association between PCOS and the selected endpoints, the high prevalence rates of these outcomes in this population were taken into consideration, and Poisson regression was used $[25,26]$. Co-variables such as age, education, oral contraceptive use and physical activity were taken into consideration in adjusting all the models. Furthermore, overweight and obesity were evaluated as co-variables when the outcomes were associated with glucose intolerance and T2DM. To construct the model, the variables were selected based on theory, on the results of the stratified analysis and using backward procedures in which those that changed the principal association by at least $10 \%$ remained in the final model. Prevalence ratios (PR) were calculated, together with their respective $95 \%$ confidence intervals (95\%CI). Goodness of fit tests were performed on the final models (Hosmer-Lemeshow and Kolmogorov-Smirnov). All the statistical analyses were conducted using the Stata software program, version 12.0 (StatsCorp, College Station, TX, USA).

\subsection{Ethical aspects}

As a multicenter study, the ELSA-Brasil study protocol was submitted to and approved by the Brazilian National Ethics Council (CONEP), as well as by the internal review boards of all the participating institutes. This study in particular was approved by the internal review board of the Collective Health Institute, Federal University of Bahia.

\section{Results}

Overall, 713 menopausal women were evaluated, with $72.9 \%$ undergoing hormone assessment and 49.4\% completing the hirsutism questionnaire. When both clinical and biochemical hyperandrogenism were taken together, i.e. response to the questionnaire and hormone analysis, data were obtained on hyperandrogenism for $85.3 \%$ of the women (608/713). The mean age of these women was 59.2 years, with $77 \%$ describing themselves as black or brown-skinned according to the Brazilian Institute of Geography and Statistics (IBGE) classification. In relation to education level, $53.4 \%$ of these women had completed high school, while $42 \%$ had graduated from university and $24.4 \%$ were postgraduates. The mean BMI of the study population was $27.8 \mathrm{~kg} / \mathrm{m}^{2}$, with $70.7 \%$ being overweight and $28 \%$ obese. T2DM was identified in $16.3 \%$ of the women and glucose intolerance in $37.8 \%$, with $72 \%$ and $8.6 \%$, respectively, currently being treated with oral hypoglycemic agents. None of the participants had testosterone levels suggestive of ovarian tumors or untreated adrenal hyperplasia.

According to the proposed criteria, $7.6 \%$ of the menopausal women were identified as having PCOS, with $7.4 \%$ fitting phenotype $1,72.2 \%$ phenotype $2,14.8 \%$ phenotype 3 and $5.6 \%$ phenotype 4 . The participants with PCOS were younger, had had fewer pregnancies and reached menopause at an earlier age. Their body weight, BMI and waist circumference were greater than those of the women without the criteria defined in this study. They had higher fasting and 2-h glucose levels, higher fasting insulin levels and, consequently, a higher HOMA index. Total cholesterol and high-density lipoprotein (HDL) cholesterol were lower and C-reactive protein was higher. Unlike SHBG, total and free testosterone levels and the free androgen index were much higher in these women than in those not classified as having PCOS. The LH/FSH ratio was also found to be much higher in the women affected by the syndrome. No significant differences were found in relation to height, to the waist-to-hip ratio or glycated hemoglobin, LDL cholesterol or arterial blood pressure (Table 1 ).

Similar results were found for the premenopausal women, with measurements being similar to those found in the postmenopausal participants and no statistically significant differences between the groups. Nevertheless, there were differences in some measurements such as total cholesterol, HDL cholesterol and the LH/FSH ratio. Tests comparing the pre- and postmenopausal women with PCOS showed these groups to be similar in the majority of the parameters evaluated (Table 1 ).

The participants with phenotypes defining PCOS not only had higher total testosterone levels and a higher HOMA index, but a gradient was found for both variables, with mean values increasing as a function of the number of criteria present $(p=0.00)$. Other characteristics related to the health of the women with PCOS also varied according to the number of criteria present, with tests for trend being significant through the strata (Table 2). The boxplots show how testosterone, HOMA and C-reactive protein varied positively with the number of criteria, whereas HDL-cholesterol varied negatively (Fig. 1).

The categorical analysis confirmed that the menopausal women identified as having PCOS in accordance with the defined criteria compared to those who did not fit into this definition tended to belong to the younger age groups of this population and were more likely to have a university degree. These women reached menopause earlier, were more likely to be overweight and had a waist circumference above the recommended size for women. As expected, IR was more common in the women with PCOS compared to those unaffected by this syndrome, particularly in the more severe categories. No significant differences were found between the two groups insofar as skin color was concerned. Likewise, the data showed no differences with respect to hypertension. Twohour glucose levels were higher, although not significantly so, in the group of women with PCOS (Table 3).

Symptoms associated with the criteria used were more common among women fitting the proposed definition of PCOS compared to the remaining participants. Nevertheless, the differences between the groups with respect to infertility rates or a previous diagnosis of PCOS were not statistically significant (Table 4).

Significant positive associations were found between PCOS and overweight, obesity and disorders of carbohydrate metabolism. In 
Table 1

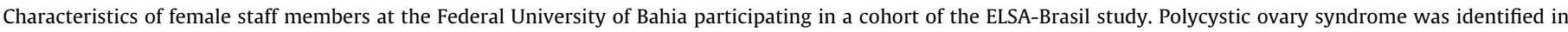

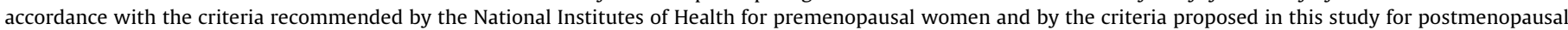
women. Salvador, Brazil, 2008-2010.

\begin{tabular}{|c|c|c|c|c|c|c|c|}
\hline \multirow[t]{4}{*}{ Variables } & \multicolumn{3}{|l|}{ Postmenopausal } & \multicolumn{4}{|l|}{ Premenopausal } \\
\hline & \multirow{2}{*}{$\begin{array}{l}\mathrm{PCOS}^{\mathrm{a}} \\
n=54\end{array}$} & \multirow{2}{*}{$\begin{array}{l}\text { No PCOS } \\
n=659\end{array}$} & \multirow{2}{*}{$\begin{array}{l}\text { Statistical test } \\
\text { Postmenopausal } \\
\text { PCOS/No PCOS }\end{array}$} & \multirow{2}{*}{$\begin{array}{l}\text { PCOS } \\
n=24\end{array}$} & \multirow{2}{*}{$\begin{array}{l}\text { No PCOS } \\
n=451\end{array}$} & \multicolumn{2}{|l|}{ Statistical test } \\
\hline & & & & & & $\begin{array}{l}\text { Premenopausal } \\
\text { PCOS/No PCOS }\end{array}$ & $\begin{array}{l}\text { PCOS pre/post- } \\
\text { menopause }\end{array}$ \\
\hline & Median (IQR) $)^{\mathrm{b}}$ & Median (IQR) & Mann-Whitney & Median (IQR) & Median (IQR) & Mann-Whitney & \\
\hline Age (years) & $58(8)$ & $59(10)$ & 0.0164 & $48((14)$ & $46(8)$ & 0.8882 & 0.0000 \\
\hline $\begin{array}{l}\text { Number of } \\
\text { pregnancies }\end{array}$ & $2(2)$ & $3(2)$ & 0.0322 & $2(2)$ & $2(2)$ & 0.5194 & 0.2284 \\
\hline Age at menopause & $45(9)$ & $48(8)$ & 0.0085 & - & - & - & - \\
\hline $\begin{array}{l}\text { Fasting glucose } \\
(\mathrm{mg} / \mathrm{dl})\end{array}$ & $111.5(25)$ & $106(17)$ & 0.0037 & $105.5(16)$ & $101(12)$ & 0.0234 & 0.0573 \\
\hline 2 -h glucose (mg/dl) & $149(73)$ & $133(62)$ & 0.0529 & $136(68)$ & $120(42)$ & 0.0103 & 0.4090 \\
\hline HbA1c (\%) & $5.5(0.9)$ & $5.3(0.8)$ & 0.2937 & $5.1(1.0)$ & $5.0(8.0)$ & 0.5787 & 0.0197 \\
\hline Insulin $(\mu \mathrm{U} / \mathrm{ml})$ & $12.2(5.6)$ & $5.5(5.9)$ & 0.0000 & $6.1(11.3)$ & $5.5(6.6)$ & 0.2055 & 0.0015 \\
\hline HOMA-IR & $3.4(2)$ & $1.5(3.7)$ & 0.0000 & $1.7(2.95)$ & $1.4(1.75)$ & 0.0982 & 0.0011 \\
\hline $\begin{array}{l}\text { Total cholesterol } \\
\text { (mg/dl) }\end{array}$ & $217(66)$ & $233(60)$ & 0.0235 & $220.5(51)$ & $216(50)$ & 0.9403 & 0.9482 \\
\hline $\begin{array}{l}\text { HDL cholesterol } \\
\qquad(\mathrm{mg} / \mathrm{dl})\end{array}$ & $56(18)$ & $63(20)$ & 0.0000 & $60.5(16.5)$ & $61(20)$ & 0.8965 & 0.0290 \\
\hline $\begin{array}{l}\text { LDL cholesterol } \\
\qquad(\mathrm{mg} / \mathrm{dl})\end{array}$ & $130(56)$ & $143(53)$ & 0.2214 & $137.5(42.5)$ & $130(45)$ & 0.8114 & 0.7576 \\
\hline $\begin{array}{l}\text { Triglycerides } \\
\text { (mg/dl) }\end{array}$ & $130.5(66)$ & $116(73)$ & 0.0955 & $98.5(94)$ & $96(67)$ & 0.9762 & 0.0299 \\
\hline $\begin{array}{l}\text { C-reactive protein } \\
(\mathrm{mg} / \mathrm{l})\end{array}$ & $2.7(4.6)$ & $2.0(2.9)$ & 0.0396 & $2.8(3.8)$ & $1.31(2.4)$ & 0.0110 & 0.8810 \\
\hline $\begin{array}{l}\text { Total testosterone } \\
(\mathrm{ng} / \mathrm{dl})\end{array}$ & $39.5(34)$ & $19(15)$ & 0.0000 & $43.5(16)$ & $23(16)$ & 0.0000 & 0.2852 \\
\hline $\begin{array}{l}\text { Free testosterone } \\
\quad(\mathrm{pg} / \mathrm{ml})\end{array}$ & $8.1(7.4)$ & $2.9(2.5)$ & 0.0000 & $6.5(3.4)$ & $3.0(2.5)$ & 0.0000 & 0.8049 \\
\hline $\begin{array}{l}\text { Free androgen } \\
\text { index }\end{array}$ & $5.3(4.8)$ & $1.5(1.6)$ & 0.0000 & $3.7(2.1)$ & $1.5(1.5)$ & 0.0000 & 0.4231 \\
\hline SHBG (nmol/l) & $32.8(23)$ & $43.5(27)$ & 0.0002 & $42.1(26)$ & $51.7(34)$ & 0.1833 & 0.0237 \\
\hline LH/FSH ratio & $0.5(0.2)$ & $0.4(0.2)$ & 0.0203 & $0.6(0.9)$ & $0.6(0.6)$ & 0.7350 & 0.0112 \\
\hline $\begin{array}{l}\text { Systolic blood } \\
\text { pressure } \\
(\mathrm{mm} \mathrm{Hg})\end{array}$ & $125.8(29.5)$ & $127(25.5)$ & 0.9696 & $119.8(26.8)$ & $115.0(22.5)$ & 0.0795 & 0.2936 \\
\hline \multirow{2}{*}{$\begin{array}{l}\text { Diastolic blood } \\
\text { pressure } \\
(\mathrm{mm} \mathrm{Hg})\end{array}$} & $78.3(12.5)$ & $77(13)$ & 0.3042 & $74.3(15.8)$ & $74.5(14)$ & 0.2096 & 0.9568 \\
\hline & Mean (SD) & Mean (SD) & Student's $t$-test & Mean (SD) & Mean (SD) & Student's $t$-test & \\
\hline Weight (kg) & $72.8(10.7)$ & $68.2(13.1)$ & 0.0122 & $72.2(13.4)$ & $64.5(13.8)$ & 0.0571 & 0.8291 \\
\hline Height (m) & $1.58(0.1)$ & $1.57(0.1)$ & 0.8969 & $1.61(0.1)$ & $1.6(0.0)$ & 0.5874 & 0.0102 \\
\hline BMI $^{\text {c }}$ (weight $/$ height $^{2}$ ) & $29.6(4.5)$ & $27.7(4.9)$ & 0.0051 & $28.1(5.3)$ & $26.1(4.9)$ & 0.0578 & 0.2042 \\
\hline Waist circumference $(\mathrm{cm})$ & $95.8(12.5)$ & $91.2(12.7)$ & 0.0114 & $92.4(12.4)$ & $86.1(11.5)$ & 0.0103 & 0.2708 \\
\hline Waist-to-hip ratio & $0.89(0.1)$ & $0.88(0.1)$ & 0.2102 & $0.88(0.0)$ & $0.84(0.0)$ & 0.0082 & 0.4083 \\
\hline
\end{tabular}

\footnotetext{
a Polycystic ovary syndrome.

b Interquartile range.

c Body mass index.
}

Table 2

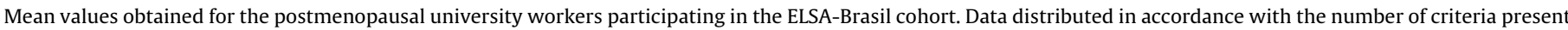
and the polycystic ovary syndrome phenotypes based on the proposed definitions $(n=713)$. Salvador, Brazil, 2008-2010.

\begin{tabular}{|c|c|c|c|c|c|}
\hline Number of criteria present & $\begin{array}{l}0 \\
n=385\end{array}$ & $\begin{array}{l}1 \\
n=274\end{array}$ & $\begin{array}{l}2 \\
n=51\end{array}$ & $\begin{array}{l}3 \\
n=3\end{array}$ & $p$-Value ${ }^{a}$ \\
\hline Age & 59.5 & 59.2 & 56.7 & 58.3 & 0.045 \\
\hline $\mathrm{BMI}^{\mathrm{b}}$ & 26.6 & 29.1 & 29.5 & 30.7 & 0.000 \\
\hline Waist circumference & 88.2 & 95.4 & 95.7 & 97.1 & 0.000 \\
\hline Fasting glucose $\mathrm{c}^{\mathrm{C}}$ & 109.1 & 124.6 & 119.5 & 108.7 & 0.000 \\
\hline Fasting insulin ${ }^{\mathrm{d}}$ & 4.0 & 11.1 & 14.6 & 10.2 & 0.000 \\
\hline HOMA $^{\mathrm{e}}$ & 1.1 & 3.4 & 4.4 & 2.7 & 0.000 \\
\hline HDL cholesterol $^{\mathrm{f}}$ & 67.9 & 59.9 & 55.2 & 53.0 & 0.000 \\
\hline Systolic blood pressure (mm Hg) & 129.0 & 129.8 & 130.5 & 121.3 & 0.582 \\
\hline Diastolic blood pressure (mm Hg) & 76.7 & 77.8 & 79.4 & 71.3 & 0.077 \\
\hline
\end{tabular}

\footnotetext{
a Cuzick's test for trend.
}

b Body mass index (weight/height ${ }^{2}$ ).

c $\mathrm{mg} / \mathrm{dl}$.

d $\mu \mathrm{U} / \mathrm{ml}$.

e Homeostasis model assessment.

f High density lipoprotein cholesterol. 

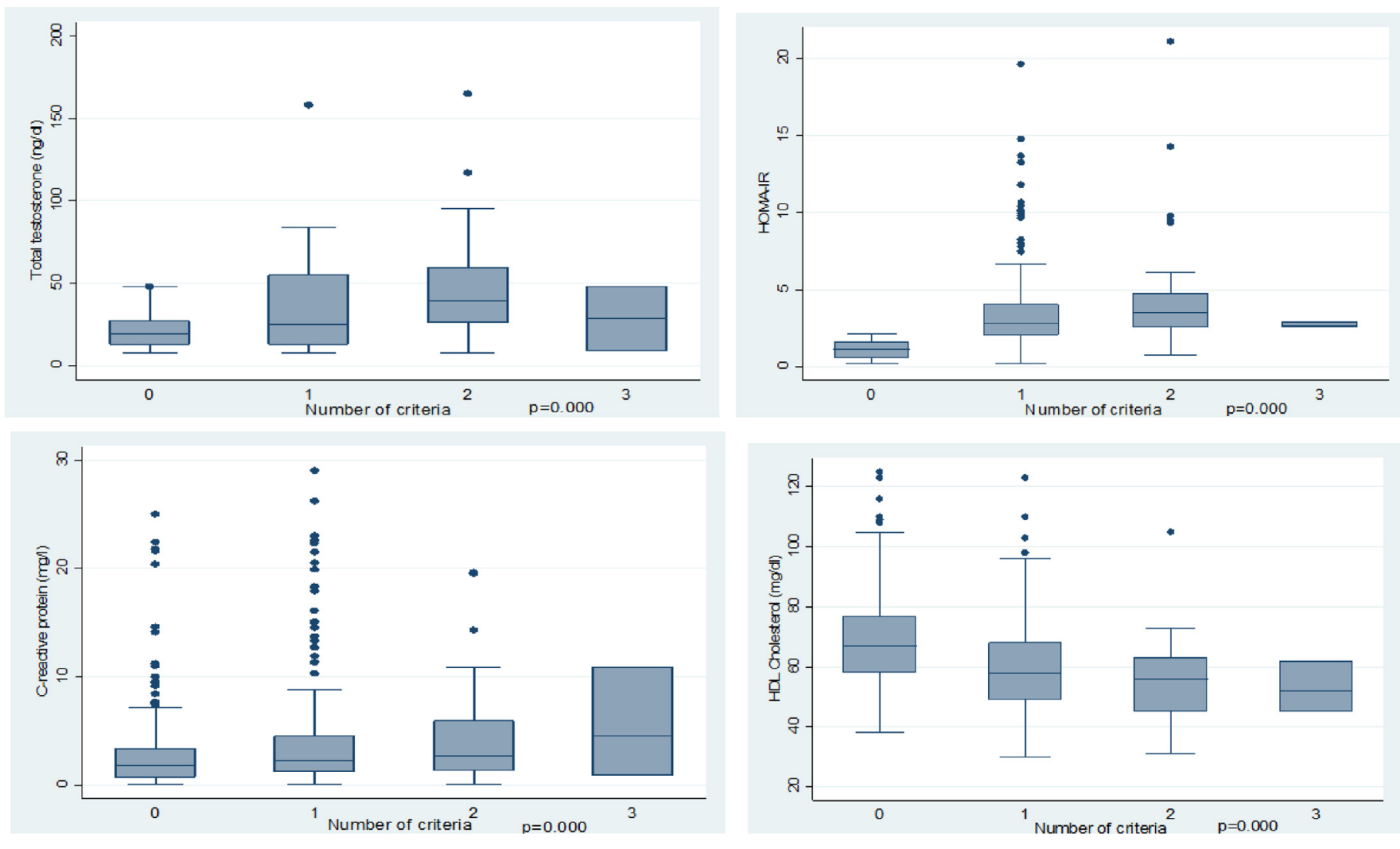

Fig. 1. Relevant health features in women with polycystic ovary syndrome according to the number of diagnostic criteria.

the stratified analysis, age significantly modified the association with overweight and with disorders of carbohydrate metabolism. None of the other co-variables evaluated changed the crude associations of the previously established limit; therefore, they were not included in the models. The past use of oral contraceptives failed to reach this limit, but was included in the models related to weight, since it was shown to be associated with these variables and because the association is theoretically plausible.

PCOS, as defined here, was positively and significantly associated with overweight following adjustment for age and the use of oral contraceptives. It was also associated with disorders of carbohydrate metabolism (glucose intolerance and T2DM) when adjusted for age, and with obesity in an unadjusted model. A positive association was found with T2DM alone; however, this did not reach statistical significance. In the group of premenopausal women, an association was found between PCOS and overweight and with T2DM alone, when controlled for age, or when analyzed together with glucose intolerance (Table 5).

\section{Discussion}

In line with the body of evidence available in the international literature on PCOS, this study proposes a set of criteria with which to identify the syndrome after menopause, with results showing that these criteria successfully selected a group of women with characteristics that were expected and strongly associated with PCOS.

Adding IR to menstrual dysfunction and hyperandrogenism, criteria already traditionally acknowledged for a diagnosis of PCOS in premenopausal women, introduced an element known to be associated with the syndrome and held by many as a crucial component in potentiating the risks associated with this condition following menopause $[17,27]$. It is not simply a case of identifying women with IR, but, rather, a sub-group of women with their own characteristics who have an additional factor, hyperandrogenism, that when associated with IR, potentiates cardiometabolic risks [28]. Data from the Study of Women's Health across the Nation (SWAN) show a strict correlation between the HOMA index and testosterone levels following adjustment for BMI [29]. The present study identified a group of women in whom IR, hyperandrogenism and excess weight are highly prevalent.

The prevalence of IR in the women identified as having the criteria for PCOS was greater. In addition, C-reactive protein levels were higher and HDL levels were lower, indicating an atherogenic profile that is associated with increased cardiovascular risk. The presence of high testosterone levels has been shown to be an important factor that is strongly associated with subclinical cardiovascular disease. In the Multi-Ethnic Study of Atherosclerosis (MESA), an evaluation conducted with menopausal women showed a positive correlation between carotid intima-media thickness and testosterone levels irrespective of insulinemia, suggesting subclinical atherosclerosis [28]. The similarities found here with the group of women of reproductive age with PCOS, selected in this study using consolidated criteria, strengthen the power of the proposed phenotypes to identify postmenopausal women with this syndrome. The differences found between these groups may be due to the older age of the menopausal women, in whom IR was more common than in the younger group. The age-dependent decline in the sensitivity of the peripheral tissues to insulin is well established, even when body weight is normal, [30]. Investigators have shown how hyperinsulinemia and IR are more relevant in older compared to younger women with PCOS, highlighting the importance of this phenomenon in the phenotype of the syndrome in older women [31]. 
Table 3

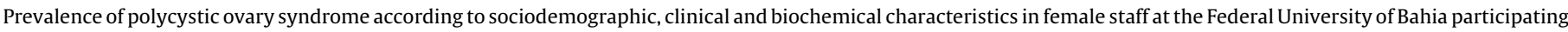
in the ELSA-Brasil cohort, identified according to the criteria proposed for a diagnosis of PCOS in menopause $(n=713)$. Salvador, Brazil, 2008-2010.

\begin{tabular}{|c|c|c|c|c|}
\hline \multirow[t]{2}{*}{ Characteristics } & \multirow[t]{2}{*}{$n=713$} & \multicolumn{2}{|c|}{ Prevalence of PCOS } & \multirow[t]{2}{*}{$p$-Value } \\
\hline & & $(\%)$ & $(95 \% \mathrm{CI})$ & \\
\hline Age & & & & 0.218 \\
\hline $35-54$ years & 175 & 9.71 & $(5.76-15.10)$ & \\
\hline 55-74 years & 538 & 6.88 & $(4.89-9.36)$ & \\
\hline Race/skin color ${ }^{\mathrm{b}}$ & & & & 0.466 \\
\hline Black & 242 & 5.4 & $(2.89-9.01)$ & \\
\hline Brown & 290 & 8.6 & $(5.66-12.46)$ & \\
\hline White & 135 & 8.9 & $(4.68-15.01)$ & \\
\hline Yellow & 11 & 9.1 & $(0.22-41.25)$ & \\
\hline Indigenous & 13 & 15.4 & $(1.92-45.45)$ & \\
\hline Years of schooling & & & & 0.039 \\
\hline$<11$ years & 109 & 2.8 & $(0.57-7.83)$ & \\
\hline$\geq 11$ years & 604 & 8.4 & $(6.35-10.95)$ & \\
\hline Age at menopause & & & & 0.008 \\
\hline$<45$ years & 209 & 12.4 & $(8.26-17.69)$ & \\
\hline 45-49 years & 218 & 6.9 & $(3.90-11.09)$ & \\
\hline$>50$ years & 266 & 4.9 & $(2.63-8.21)$ & \\
\hline $\mathrm{BMI}^{\mathrm{c}}$ & & & & 0.007 \\
\hline$<25$ & 209 & 2.87 & $(1.06-6.14)$ & \\
\hline $25-30$ & 304 & 8.88 & $(5.94-12.66)$ & \\
\hline$\geq 30$ & 199 & 10.55 & $(6.65-15.68)$ & \\
\hline Waist circumference & & & & 0.017 \\
\hline$\geq 88 \mathrm{~cm}$ & 280 & 4.64 & $(6.88-12.63)$ & \\
\hline$<88 \mathrm{~cm}$ & 433 & 9.47 & $(2.49-7.81)$ & \\
\hline 2-h glucose $\mathrm{d}^{\mathrm{d}}$ & & & & 0.080 \\
\hline$\geq 140 \mathrm{mg} / \mathrm{dl}$ & 295 & 8.8 & $(5.84-12.65)$ & \\
\hline$<140 \mathrm{mg} / \mathrm{dl}$ & 341 & 5.3 & $(3.16-8.21)$ & \\
\hline Insulin resistance $\mathrm{e}^{\mathrm{e}}$ & & & & $0.000^{f}$ \\
\hline Normal $(<2.0)$ & 427 & 0.7 & $(0.15-2.04)$ & \\
\hline Borderline (2.0-2.2) & & - & - & \\
\hline Moderate (2.2-3.0) & 90 & 18.9 & $(11.41-28.51)$ & \\
\hline Severe $(\geq 3.0)$ & 165 & 20.6 & $(14.71-27.59)$ & \\
\hline Blood pressure & & & & 0.704 \\
\hline Normal & 223 & 7.2 & $(4.16-11.39)$ & \\
\hline Prehypertension & 286 & 7.0 & $(4.32-10.59)$ & \\
\hline Hypertension & 202 & 8.9 & $(5.37-13.71)$ & \\
\hline
\end{tabular}

a Polycystic ovary syndrome.

b Accord ing to the classification defined by the Brazilian Institute of Geography and Statistics (IBGE).

c Body mass index (weight/height ${ }^{2}$ ).

d Measured $2 \mathrm{~h}$ following a $75 \mathrm{~g}$ oral glucose load, in $\mathrm{mg} / \mathrm{dl}$.

e HOMA (homeostasis model assessment).

f Fisher's exact test.

The questionnaire administered at baseline in the ELSA-Brasil study failed to obtain data on previous dysfunctional uterine bleeding, a common occurrence in anovulatory women, thus reducing the possibility of identifying participants with menstrual dysfunction. Identifying these cases more accurately would probably reduce the proportion of participants with the other phenotypes. It is important to emphasize possible limitations in the data obtained on the menstrual characteristics of these postmenopausal women as a result of possible recall bias, once these women had not been menstruating for at least one year at the beginning of the study. Since it was not possible to perform pelvic ultrasonography, the inclusion of ovarian morphology in the proposed criteria was not cogitated. It is known that, even after menopause, the ovaries of women with PCOS may retain a polycystic appearance and that women with this characteristic tend to have a hyperandrogenic hormone profile [12].

The present data confirm that the severity of PCOS is cumulative, i.e. the more diagnostic criteria present, the greater the associations with metabolic or cardiovascular health risk factors [13,14]. PCOS is a heterogenic syndrome with various signs, symptoms and hormonal markers that classify its severity based on the number of components presented [3].

The criteria proposed here were able to identify women with PCOS and can be used in population-based studies. These
Table 4

Prevalence of components of the proposed criteria for the identification of polycystic ovary syndrome in the menopause in female staff members of the Federal University of Bahia participating in the ELSA-Brasil cohort $(n=713)$. Salvador, Brazil. 2008-2010.

\begin{tabular}{llcl}
\hline Components & PCOS (\%) & No PCOS (\%) & $p$-Value \\
\hline Oligomenorrhea $^{\mathrm{a}}$ & 27.8 & 2.5 & 0.000 \\
Hirsutism $^{\mathrm{b}}$ & 80.0 & 15.1 & 0.000 \\
Total testosterone $^{\mathrm{c}}$ & 28.3 & 2.5 & 0.000 \\
Free testosterone $^{\mathrm{c}}$ & 41.3 & 1.5 & 0.000 \\
Free androgen index $_{\text {Insulin resistance }}^{\mathrm{d}}$ & 34.8 & 2.1 & 0.000 \\
\hline
\end{tabular}

a Intermenstrual interval $\geq 35$ days.

b $\geq 5$ points on the hirsutism questionnaire.

c $\geq 95$ th percentile of women considered healthy.

d HOMA (homeostasis model assessment) $\geq 75$ th percentile of non-obese and non-diabetic women.

women had an increased risk of excess weight and disorders of carbohydrate metabolism, with a potential increased risk of cardiovascular disease, the major endpoint in the evaluation of PCOS in menopausal women. The longitudinal follow-up of these participants of the ELSA-Brasil study will allow these criteria to be consolidated, adding to what is already known on the health of this group of women. 
Table 5

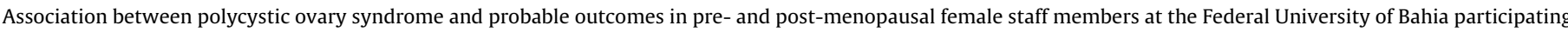
in the ELSA-Brasil cohort $(n=713)$. Salvador, Brazil, 2008-2010.

\begin{tabular}{|c|c|c|c|c|c|c|}
\hline \multirow[t]{2}{*}{ Outcomes } & \multicolumn{3}{|c|}{ Post-menopausal women } & \multicolumn{3}{|c|}{ Pre-menopausal women } \\
\hline & Prevalence (\%) & PR & $95 \% \mathrm{CI}$ & Prevalence (\%) & PR & $95 \% \mathrm{CI}$ \\
\hline $\mathrm{BMI}^{\mathrm{a}} \geq 25 \mathrm{~kg} / \mathrm{m}^{2}$ & 6.80 & & & 6.56 & & \\
\hline Crude & & 1.29 & $(1.16-1.43)$ & & 1.32 & $(0.98-1.77)$ \\
\hline Adjusted $^{\mathrm{b}}$ & & 1.31 & $(1.18-1.46)$ & & 1.34 & $(1.02-1.77)$ \\
\hline $\mathrm{BMI}^{\mathrm{a}} \geq 30 \mathrm{~kg} / \mathrm{m}^{2}$ & 9.09 & & & 7.84 & & \\
\hline Crude & & 1.44 & $(1.01-2.06)$ & & 1.60 & $(0.88-2.89)$ \\
\hline Adjusted $^{\mathrm{b}}$ & & 1.41 & $(0.96-2.06)$ & & 1.64 & $(0.91-2.97)$ \\
\hline Glucose intolerance and $\mathrm{T}^{2} \mathrm{DM}^{\mathrm{c}}$ & 6.71 & & & 9.02 & & \\
\hline Crude & & 1.30 & $(1.03-1.65)$ & & 1.74 & $(1.13-2.66)$ \\
\hline Adjusted $^{\mathrm{d}}$ & & 1.42 & $(1.13-1.79)$ & & 1.78 & $(1.16-2.72)$ \\
\hline T2DM & 11.21 & & & 4.17 & & \\
\hline Crude & & 1.41 & $(0.83-2.39)$ & & 0.82 & $(0.12-5.81)$ \\
\hline Adjusted $^{\mathrm{d}}$ & & 1.58 & $(0.93-2.67)$ & & 1.01 & $(0.14-7.22)$ \\
\hline
\end{tabular}

a Body mass index (weight/height ${ }^{2}$ ).

b Adjusted for age and the use of oral contraceptives.

c Type 2 diabetes mellitus.

d Adjusted for age.

\section{Contributors}

LG worked on the conception of the study and on the protocol, coordinated and supervised the fieldwork, carried out the statistical analysis and interpretation of data, and drafted the manuscript. EMLA contributed to the conception of the study, to the analysis, and the discussion of the results, and helped draft the manuscript. MCCA contributed substantially with the statistical analysis and interpretation of data. All three authors read and approved the final manuscript.

\section{Competing interest}

The authors declare no conflict of interest.

\section{Funding}

The ELSA-Brasil baseline study was supported by the Brazilian Ministry of Health (Science and Technology Department) and the Brazilian Ministry of Science and Technology (Funding Source for Studies and Projects and the National Research Council, CNPq) (grant number $01060212.00 \mathrm{BA}$ ). This ancillary study received financial support from the National Research Council (CNPq) according to the public document MCT-CNPq number 14/2009 and grant number 474956/2009-1.

\section{Ethical approval}

This is a supplementary study of a greater research funded by the Health Ministry of Brazil called ELSA-Brasil (Estudo Longitudinal de Saúde do Adulto - The Brazilian Longitudinal Study of Adult Health). Which is a cohort study of 15,105 civil servants from 5 universities and 1 research institute located in different regions of Brazil: the federal universities of Bahia, Espirito Santo, Minas Gerais, and Rio Grande do Sul; the University of Sao Paulo; and the Oswaldo Cruz Foundation in Rio de Janeiro. The main study objectives of ELSA-Brasil are to investigate the incidence and progression of diabetes and cardiovascular diseases and their biologic, behavioral, environmental, occupational, psychological, and social factors. Both upstream (social patterns and structures that shape people's chances to be healthy) and downstream (individual characteristics) risk factors will be assessed for their possible associations with subclinical and clinical outcomes. Because it is a multicenter study, ELSA-Brasil's research protocol was approved not only by the ethics committee of each institution but also by the National Research Ethics Committee. In addition to the usual informed consent, participants are also asked to provide signed consent.

\section{Acknowledgements}

The authors would like to thank the support of the Brazilian Ministry of Health (Science and Technology Department), the Brazilian Ministry of Science and Technology and the National Research Council (CNPq). This study was conducted at the Federal University of Bahia's Research Center for the Brazilian Longitudinal Study of Adult Health (ELSA-Brasil), Salvador, Bahia, Brazil.

The authors would also like to thank Dr. Moyses Szklo for his useful and wise comments, Dr. Eduardo Mota for his academic and institutional input, and the entire staff at the ELSA Research Center in Bahia for their unconditional support.

\section{References}

[1] Diamanti-Kandarakis E, Kouli CR, Bergiele AT, et al. A survey of the polycystic ovary syndrome in the Greek island of Lesbos: hormonal and metabolic profile. J Clin Endocrinol Metab 1999;84:4006-11.

[2] Tehrani FR, Simbar M, Tohidi M, Hosseinpanah F, Azizi F. The prevalence of polycystic ovary syndrome in a community sample of Iranian population: Iranian PCOS prevalence study. Reprod Biol Endocrinol 2011;9:39.

[3] Amsterdam ESHRE/ASRM-Sponsored 3rd PCOS Consensus Workshop Group. Consensus on women's health aspects of polycystic ovary syndrome (PCOS). Hum Reprod 2012;27:14-24

[4] Talbott EO, Guzick DS, Sutton-Tyrrell K, et al. Evidence for association between polycystic ovary syndrome and premature carotid atherosclerosis in middleaged women. Arterioscler Thromb Vasc Biol 2000;20:2414-21.

[5] Margolin E, Zhornitzki T, Kopernik G, Kogan S, Schattner A, Knobler H. Polycystic ovary syndrome in post-menopausal women - marker of the metabolic syndrome. Maturitas 2005;50:331-6.

[6] Carmina E, Campagna AM, Lobo RA. Emergence of ovulatory cycles with aging in women with polycystic ovary syndrome (PCOS) alters the trajectory of cardiovascular and metabolic risk factors. Hum Reprod 2013;28:2245-52.

[7] Pasquali R, Gambineri A. Polycystic ovary syndrome: a multifaceted disease from adolescence to adult age. Ann NY Acad Sci 2006;1092:158-74

[8] Winters SJ, Talbott E, Guzick DS, Zborowski J, McHugh KP. Serum testosterone levels decrease in middle age in women with the polycystic ovary syndrome. Fertil Steril 2000;73:724-9.

[9] Vlachopoulos C, Ioakeimidis N, Terentes-Printzios D, et al. Plasma total testosterone and incident cardiovascular events in hypertensive patients. Am J Hypertens 2013;26:373-81.

[10] Birdsall MA, Farquhar CM, White HD. Association between polycystic ovaries and extent of coronary artery disease in women having cardiac catheterization. Ann Intern Med 1997:126:32-5.

[11] Azevedo GD, Duarte JM, Souza MO, Costa-e-Silva TD, Soares EM, Maranhão TM. Menstrual cycle irregularity as a marker of cardiovascular risk factors at postmenopausal years. Arq Bras Endocrinol Metabol 2006;50:876-83.

[12] Birdsall MA, Farquhar CM. Polycystic ovaries in pre and post-menopausal women. Clin Endocrinol (Oxf) 1996;44:269-76. 
[13] Krentz AJ, von Mühlen D, Barrett-Connor E. Searching for polycystic ovary syndrome in postmenopausal women: evidence of a dose-effect association with prevalent cardiovascular disease. Menopause 2007;14:284-92.

[14] Krentz AJ, von Mühlen D, Barrett-Connor E. Adipocytokine profiles in a putative novel postmenopausal polycystic ovary syndrome (PCOS) phenotype parallel those in premenopausal PCOS: the Rancho Bernardo Study. Metabolism 2012;61:1238-41.

[15] Azziz R, Carmina E, Dewailly D, et al. Positions statement: criteria for defining polycystic ovary syndrome as a predominantly hyperandrogenic syndrome: an Androgen Excess Society guideline. J Clin Endocrinol Metab 2006;91:4237-45.

[16] Dewailly D, Catteau-Jonard S, Reyss AC, Leroy M, Pigny P. Oligoanovulation with polycystic ovaries but not overt hyperandrogenism. J Clin Endocrinol Metab 2006;91:3922-7.

[17] Conway G, Dewailly D, Diamanti-Kandarakis E, et al. The polycystic ovary syndrome: a position statement from the European Society of Endocrinology. Eur J Endocrinol 2014;171:P1-29.

[18] Gabrielli L, Aquino EM. Polycystic ovary syndrome in Salvador. Brazil: a prevalence study in primary healthcare. Reprod Biol Endocrinol 2012:10:96.

[19] Aquino EM, Barreto SM, Bensenor IM, et al. Brazilian Longitudinal Study of Adult Health (ELSA-Brasil): objectives and design. Am J Epidemiol 2012;175: 315-24.

[20] Gabrielli L, Aquino EM. A simplified questionnaire for self-assessment of hirsutism in population-based studies. Eur J Endocrinol 2015;172(April (4)):451-9 [Epub 2015 January 12].

[21] Matthews DR, Hosker JP, Rudenski AS, Naylor BA, Treacher DF, Turner RC. Homeostasis model assessment: insulin resistance and beta-cell function from fasting plasma glucose and insulin concentrations in man. Diabetologia $1985 ; 28: 412-9$
[22] Garmendia ML, Lera L, Sánchez H, Uauy R, Albala C. Homeostasis model assessment (HOMA) values in Chilean elderly subjects. Rev Med Chil 2009; 137:1409-16.

[23] American Diabetes Association. Standards of medical care in diabetes - 2012. Diabetes Care 2012;35(Suppl. 1):S11-63.

[24] World Health Organization. Definition and diagnosis of diabetes mellitus and intermediate hyperglycaemia. In: Report of a WHO/IDF consultation. Geneva: World Health Organization; 2006.

[25] Barros AJ, Hirakata VN. Alternatives for logistic regression in cross-sectional studies: an empirical comparison of models that directly estimate prevalence ratios. BMC Med Res Methodol 2003;3:21.

[26] Petersen MR, Deddens JA. A comparison of two methods for estimating prevalence ratios. BMC Med Res Methodol 2008;8:9.

[27] Azziz R, Carmina E, Dewailly D, et al. The Androgen Excess and PCOS Society criteria for the polycystic ovary syndrome: the complete task force report. Fertil Steril 2009;91:456-88.

[28] Ouyang P, Vaidya D, Dobs A, et al. Sex hormone levels and subclinical atherosclerosis in postmenopausal women: the Multi-Ethnic Study of Atherosclerosis. Atherosclerosis 2009;204:255-61.

[29] Sutton-Tyrrell K, Wildman RP, Matthews KA, et al. Sex-hormone-binding globulin and the free androgen index are related to cardiovascular risk factors in multiethnic premenopausal and perimenopausal women enrolled in the Study of Women Across the Nation (SWAN). Circulation 2005;111:1242-9.

[30] Rowe JW, Minaker KL, Pallotta JA, Flier JS. Characterization of the insulin resistance of aging. J Clin Invest 1983;71:1581-7.

[31] Liang SJ, Hsu CS, Tzeng CR, Chen CH, Hsu MI. Clinical and biochemical presentation of polycystic ovary syndrome in women between the ages of 20 and 40 . Hum Reprod 2011;26:3443-9. 